\title{
Norois
}

Environnement, aménagement, société

\section{Un lieu en controverse : une controverse qui fait $\operatorname{lieu}(\mathrm{x})$}

Place, space and controversy

Valérie November, Cristina D’Alessandro-Scarpari et Élisabeth Remy

\section{(2) OpenEdition}

\section{Journals}

Édition électronique

URL : http://journals.openedition.org/norois/799

DOI : $10.4000 /$ norois. 799

ISBN : 978-2-7535-1541-3

ISSN : $1760-8546$

Éditeur

Presses universitaires de Rennes

Édition imprimée

Date de publication : 1 décembre 2004

Pagination : $91-102$

ISBN : 978-2-7535-0081-5

ISSN : 0029-182X

\section{Référence électronique}

Valérie November, Cristina D’Alessandro-Scarpari et Élisabeth Remy, « Un lieu en controverse : une controverse qui fait lieu(x)», Norois [En ligne], 193 | 2004/4, mis en ligne le 18 août 2008, consulté le 01 mai 2019. URL : http://journals.openedition.org/norois/799 ; DOI : 10.4000/norois.799

Ce document a été généré automatiquement le 1 mai 2019.

(c) Tous droits réservés 


\section{Un lieu en controverse : une controverse qui fait lieu(x)}

Place, space and controversy

Valérie November, Cristina D'Alessandro-Scarpari et Élisabeth Remy

\section{NOTE DE L'ÉDITEUR}

Cet article a été reçu le 17 juin 2004 et définitivement accepté le 2 novembre 2004.

\section{Introduction}

1 Si les controverses socio-techniques ont déjà fait l'objet de nombreuses publications scientifiques (Wynne 1997, Limoges 1993), il est frappant de constater que ce sujet d'étude est peu abordé, en tant que tel, par les géographes. Or, la composante spatiale est pourtant bien présente dans ce genre de situation, car la controverse peut concerner des espaces qui vont se trouver pris dans le débat. La question que nous souhaitons aborder dans cet article est de clarifier le rôle que joue la composante spatiale dans les controverses : n'est-elle qu'un prétexte argumentatif ou joue-t-elle un rôle effectif dans le déroulement des controverses? Autrement dit, il convient alors de s'interroger sur la manière dont géographes et sociologues peuvent appréhender la dimension géographique des controverses.

2 Pour répondre à une telle interrogation, les controverses autour d'installations techniques apparaissent comme un objet privilégié. Elles permettent à l'analyste d'observer comment des acteurs, y compris et surtout des non-techniciens, engagent le débat (prennent la parole, argumentent, critiquent, etc.) à propos d'options et de thèmes souvent très ardus sur le plan scientifique, juridique ou technique, et de tester la présence éventuelle des éléments spatiaux et leurs modalités d'émergence. Dans ce texte, 
nous suivrons plus précisément la manière dont une ligne à haute tension (LHT dans la suite de cet article) a pu être mise en débat public par des non spécialistes ${ }^{1}$.

Certes, il existe un courant de recherche, intitulé "géographie des conflits", qui s'est intéressé en priorité aux conflits d'aménagement ou aux conflits environnementaux (Lecourt, 2003). Parmi les principaux travaux menés, nous pouvons signaler ceux de B. Charlier (1999), de J. Ollivro (1994) et de G. Faburel (2003) portant respectivement sur des conflits liés à la construction d'infrastructures de transports terrestres, du TGV Méditerranée et les nuisances sonores provoqués par les avions. Cependant, ils s'inscrivent avant tout dans une approche de géographie régionale et aménagiste. Pour notre part, notre approche se fonde sur les apports de l'anthropologie des sciences en matière de controverses. Celle-ci a contribué à remettre en cause le partage entre les faits et les valeurs, partage dont elle a fait apparaître qu'il pouvait nuire à la compréhension des sciences et consécutivement à celle du sens commun (Latour, 1989, 1988; Callon et al., 2001). Suivant la démarche préconisée par ces auteurs, nous prendrons soin, pour décrire la manière dont des opinions publiques se forment au cours de la controverse, de traiter avec le même sérieux les connaissances réputées basées sur un savoir technique et les avis émis par des non-spécialistes. Il ne s'agira donc pas ici de révéler ce qui, dans la controverse, dépasse les personnes, ni même de dégager le sens caché qui guide leurs actions ou leurs paroles, mais bien de comprendre comment la controverse s'effectue et quelles sont les contraintes que les personnes ont dû prendre en compte, dans la situation où elles se trouvaient insérées, pour rendre leurs critiques ou leurs justifications acceptables par d'autres (Boltanski, 1990). Bref, notre tâche consistera à essayer de comprendre la dynamique de la controverse, en observant comment les acteurs s'y prennent pour montrer aux autres que leurs actions et leurs propos ont un sens et que la position qu'ils défendent dépasse leur simple cas particulier.

Dans cette perspective, trois conditions nous paraissent nécessaires pour qu'une notion de géographie puisse s'appliquer à l'analyse de la controverse : aller au-delà du couple nature/culture et de la séparation entre les faits et les valeurs partant du principe que les connaissances scientifiques font partie du problème et qu'il n'est guère possible de faire une sociologie de l'environnement sans intégrer celle des sciences ${ }^{2}$ (Latour et al., 1991); rendre compte de l'hétérogénéité des «mises en connexions» qui se produisent en continu lors des discussions ; avoir la souplesse de rendre compte des délimitations faites, en cours et à venir, telles qu'elles se font et se défont au cours de la controverse.

Une revue de la littérature géographique, bien que partielle, donne rapidement l'idée que les notions d'espace, de territoire, de milieu et, dans une moindre mesure, celle de lieu sont des notions polysémiques tant du point de vue de leur définition que de leur utilisation. Il suffit pour s'en convaincre de lire le dictionnaire de la géographie et de l'espace des sociétés (Lévy et Lussault, 2003). Notre projet n'est pas de définir le «bon » terme pour analyser les controverses, il est au contraire de soumettre l'une de ces notions, celle de lieu, à l'épreuve d'une controverse, et nous expliciterons dans le corps du texte les raisons de ce choix. Nous voudrions donc aboutir, par ce biais, à déceler les dimensions géographiques des controverses que l'on rencontre fréquemment dans l'étude des problèmes environnementaux et des risques. Autrement dit, nous chercherons à montrer la capacité d'une controverse à «faire » lieux et symétriquement ce que ces lieux « font faire » en situation de controverse. 


\section{Une ligne sous haute tension}

\section{L'entrée en controverse}

Pour améliorer une qualité de service qu'elle juge insuffisante, EDF envisage de créer un poste source $63 / 20 \mathrm{kV}$ (poste de transformation ou plus simplement «transfo » dans le langage courant) près de Balbec, un petit bourg réputé au plan touristique ${ }^{3}$. Le nouveau poste sera alimenté à partir du poste de Villetôt, une autre commune distante d'une dizaine de kilomètres, par l'intermédiaire d'une LHT de $63 \mathrm{kV}$ (fig. 1). Il s'agira donc à la fois d'implanter un poste source et de construire une ligne. Or, ce double projet va rapidement susciter un débat au plan local: d'un côté, des élus vont s'affronter pour obtenir l'implantation du poste source sur leur commune (celui-ci étant synonyme pour eux de taxe professionnelle importante); de l'autre côté, va se constituer un comité regroupant des riverains et plusieurs associations locales de défense de l'environnement et du patrimoine, hostiles à la défiguration du paysage par la LHT. Une fois que le comité de défense a réussi à mettre la LHT en débat, les différents opposants sont amenés à acquérir des savoirs et à se documenter pour tenter de négocier en face des experts d'EDF certaines options techniques et l'application de certaines procédures. C'est ainsi que pour qui veut participer au débat public et faire entendre sa voix, apprivoiser la technique devient à la fois une exigence et un recours. Les travaux de J. Lolive (1999) sur le tracé du TGV Méditerranée l'ont par exemple également montré.

7 De quelle controverse s'agit-il ? L'installation de la ligne à haute tension fait surgir un débat public, dans lequel les non-spécialistes arrivent à jouer un rôle de première importance, en apprivoisant la technique concernant l'objet qui fait débat. Pour espérer obtenir la révision du projet d'EDF et la mise à l'étude de toutes les alternatives possibles, le comité de défense doit se livrer à deux tâches complémentaires : il lui faut se doter d'un public dont il sera le porte-parole; il lui faut accumuler des connaissances techniques pour équilibrer la discussion avec les experts. La première tâche se traduit par un effort pour contacter et mobiliser le maximum d'habitants des environs, surtout les agriculteurs. C'est ainsi qu'en septembre 1992, une pétition recueille plus de 600 signatures. Celle-ci permet la production du public en tant que tel et donne ainsi au comité les moyens de se faire le porte-parole d'une cause fondée.

Une fois les contours d'un public esquissés, l'un des enjeux de la controverse devient l'organisation d'un forum de discussion commun, c'est-à-dire d'épreuves permettant aux différents porte-paroles de confronter directement leurs points de vue, au lieu de s'exprimer sousforme de monologue depuis des univers différents. Deux mois plus tard, le comité sollicite les candidats aux élections législatives de la troisième circonscription du département en leur envoyant un petit questionnaire accompagné d'une lettre. Celle-ci rappelle aux candidats les actions menées par le comité et les raisons de son inquiétude face à la LHT : la gêne qu'elle occasionnerait pour les agriculteurs, le tourisme, la richesse du patrimoine. Elle présente ensuite la solution envisagée par le comité, c'est-à-dire l'enfouissement de la ligne. Enfin, le comité demande aux candidats de répondre au questionnaire joint. Comme l'indique le dossier constitué par le comité à la suite de cet envoi, l'opération recueille les réponses des principaux candidats, toutes couleurs politiques confondues. 
9 Avec ce deuxième effort de mobilisation, on comprend que pour devenir des interlocuteurs sérieux des pouvoirs publics et d'EDF, et avoir le droit de discuter ensemble, il faut tout à la fois représenter quelque chose (et donc se construire un public et solliciter le soutien des élus) et accumuler des ressources et des savoirs aussi bien par rapport à la procédure juridique que par rapport aux aspects techniques du projet. L'opinion et la technique sont ici intimement mêlées, et c'est pourquoi les dispositifs où l'opinion se manifeste, dépassent très largement le cadre des seuls pétitions ou manifestations. Les modalités de la prise de parole dépendent en effet non seulement de la capacité à construire son public et à représenter l'intérêt général (comme dans les théories classiques de la représentation) mais aussi des moyens disponibles pour s'informer et se former au niveau technique et juridique.

Comme on peut le constater ici, des éléments spatiaux (emplacement de la ligne, paysage, enfouissement de la ligne, patrimoine, etc.) font partie de la constellation d'arguments invoqués par le comité pour solidifier son propos. Ceux-ci jouent un rôle important dans la constitution des avis qui prennent forme et se stabilisent au cours de cette controverse.

11 Premier constat: la dimension géographique n'est pas complètement absente de cette controverse socio-technique mais celle-ci apparaît de manière implicite, jamais centrale dans l'analyse menée. Pour aller plus loin dans l'explicitation de cette dimension présente dans la controverse et dépasser cette géographie spontanée mêlant indistinctement les notions d'espace, de territoire ou de paysage, il nous semble important de mettre certains concepts géographiques à l'épreuve du cahier des charges que nous nous sommes fixés pour tenter de mieux appréhender la dimension spatiale de la controverse ${ }^{4}$.

\section{La fabrique du lieu}

12 De quoi discute-t-on dans une controverse socio-technique? Quels arguments liés à l'espace sont convoqués? Sur quel mode ? Et comment en rendre compte pour satisfaire à la fois les exigences de la sociologie et de la géographie intéressées par l'analyse des controverses? En guise d'essai de conceptualisation, la notion de lieu a retenu notre attention; elle sera testée pour tenter de définir les processus de reconfiguration spatiale que nous voyons se déployer dans une controverse ${ }^{5}$. Pour ce faire, il est nécessaire de donner plusieurs exemples empiriques pour saisir la nature des discussions produites en situation controversée.

\section{L'exemple du centre de gravité des charges ou la convocation dans la controverse d'arguments liés à «l'espace »}

13 Les opposants ont réussi à inaugurer avec EDF un espace de discussion commun. Le comité espère pouvoir négocier sur tous les plans. EDF, de son côté, s'en tient à sa politique : informer, écouter les opposants, tout en considérant que le tracé de la ligne est une chose acquise. À l'objet instable et mal défini du comité s'oppose un objet déjà formalisé dont le seul point indéfini concerne l'implantation du poste source. C'est ce point indéfini dont vont se saisir les non-spécialistes pour s'engouffrer en force dans la question technique, faire entendre leur voix et tenter de défaire le projet initial d'EDF.

Très schématiquement, l'on peut dire que le calcul du centre de gravité des charges est ce qui permet de déterminer l'endroit où il serait utile d'implanter un point d'injection, 
c'est-à-dire un poste source. Ce calcul est donc un élément tout à fait stratégique du point de vue des riverains et des élus. C'est pourquoi deux acteurs, au départ non-spécialistes, vont s'atteler à recalculer le centre de gravité, dans un but différent : le maire de Brissol pour prouver que l'emplacement du poste source doit se faire sur sa commune et un membre de l'association régionale pour, au contraire, remettre en cause la totalité du tracé (fig. 1).

Figure 1 : Carte schématique de localisation Schematic location map

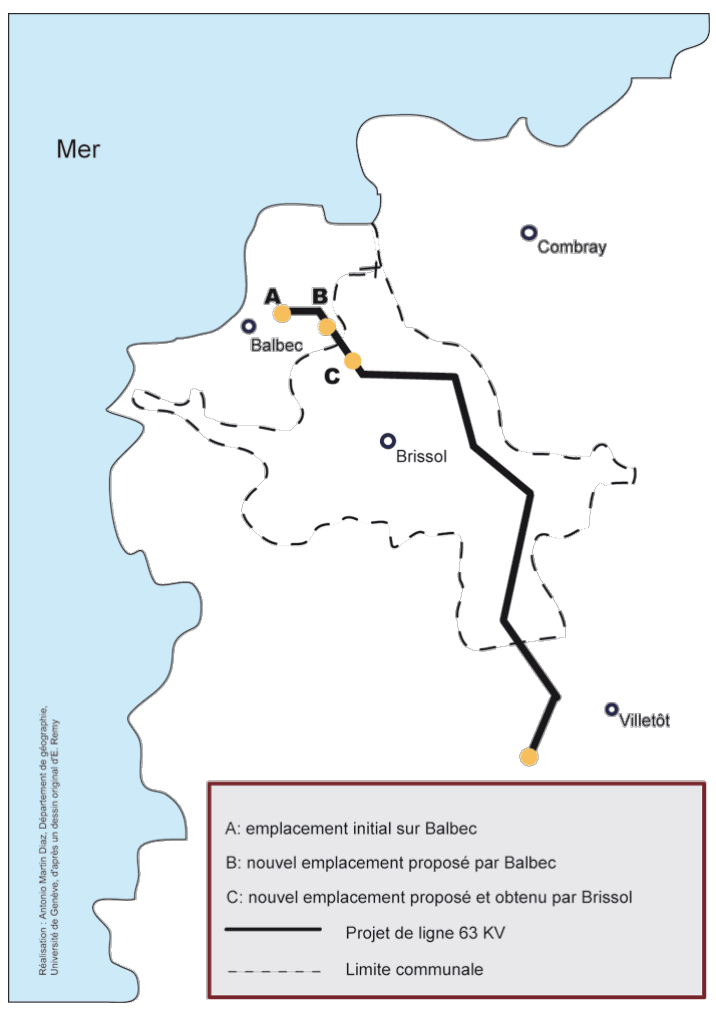

Pour déterminer le centre de gravité, le membre de l'association s'est arrangé avec un ami qui lui a fourni un certain nombre d'informations " en douce ». Il a ainsi pu réaliser un certain nombre de calculs, les reporter sur une carte et conclure finalement que le poste devait être reculé et la ligne passer plus à l'est: «On a pensé que le centre de gravité pouvait être calculé d'après l'argent versé aux communes qui est proportionnel aux consommations. La commune de Combray avait le tiers et on l'avait traduit sur une carte... partant de là, on s'est dit que les appels étant le plus important à l'est du canton de Combray, on pouvait imaginer quelque chose comme ça. D'après nous le centre de gravité est bien plus à l'est. » Le déplacement de la ligne vers l'est aurait pour conséquences, selon l'association, de permettre des économies (notamment en évitant l'aller-retour du poste source) et de mieux préserver l'environnement. Pour l'association, une modification du tracé s'impose donc, dans la mesure où «le centre de gravité est variable ». Telle est la conception de l'ouvrage qu'elle défend.

De son côté, le maire aboutit à des calculs très différents. En juillet 1991, il écrit une lettre à EDF où il démontre que le centre de gravité des charges ne doit pas être situé à Balbec mais à Brissol : « Le calcul fait d'après les surtaxes municipales versées en 1990 pour ces communes donne le centre de gravité plutôt à Brissol entre les communes de Combray, Perssay-Combray (82 343 euros) et les autres communes (71 976 euros). Brissol avec 19 
408 euros se situe par conséquent bien au centre de gravité des consommations donc des charges puisque les taxes municipales sont calculées d'après les consommations.» Appartenant au syndicat d'électrification, le maire a obtenu ses informations de base plus facilement que le comité, comme il l'explique : «J'ai fait une étude à partir des surtaxes qui sont payées par les usagers pour l'ensemble du syndicat d'électrification de la région de Combray et on s'est aperçu que globalement le centre de gravité des charges tombait à peu près à Brissol, secteur qui couvre à peu près la zone alimentée par le futur poste. La situation géographique la plus équilibrée était effectivement sur la commune de Brissol. Les gens d'EDF ont reconnu que mon étude était judicieuse. » Il est tout à fait remarquable que bien que l'association de défense et le maire se soient appuyés sur les mêmes données, à savoir les taxes d'électrification, les résultats auxquels ils sont parvenus sont divergents. À partir des mêmes données, deux séries de calculs tracent deux ouvrages différents : pour le comité, la ligne doit passer plus à l'est ; pour le maire, la ligne n'a pas à bouger et le poste doit s'implanter sur sa commune. La représentation cartographique sert d'outil de visualisation afin de montrer que l'espace et ses caractéristiques justifient le déplacement à l'est (un déplacement non casuel) du centre de gravité des charges : en effet c'est la mise en relation de tout un ensemble d'éléments hétérogènes qui " décide " de l'emplacement du site en question. En cela, le calcul relatif au centre de gravité des charges contribue à proposer une nouvelle configuration de la LHT et de "l'espace " qu'elle traverse.

On ne peut donc pas réduire le discours des acteurs à des jeux de pouvoirs qui s'effectueraient en laissant de côté la technique : l'ouvrage et son juste tracé constituent bel et bien le centre de la discussion. C'est pourquoi chaque acteur, s'il veut faire entendre sa voix et défendre ses intérêts, doit faire l'effort de se procurer des données et de se plonger dans des calculs sérieux et convaincants pour les autres. De ce fait, la question ne saurait être pour nous de départager les calculs « intéressés » et les calculs « objectifs » et "désintéressés » : elle est plutôt de comprendre comment une définition commune du centre de gravité et conséquemment du projet final, satisfaisant les calculs techniques et politiques des uns et des autres, arrive progressivement à se stabiliser.

Cette stabilisation est sans doute rendue plus facile par le fait que les experts d'EDF, de leur côté, n'ont pas une conception très rigide ni très précise de l'endroit où se situe le centre de gravité. Pour les ingénieurs que nous avons rencontrés, un centre de gravité des charges, ce n'est rien de plus qu'une zone techniquement judicieuse pour implanter le point d'injection : «Ce n'est pas un point sur la carte, c'est une surface bien évidemment parce qu'on peut très bien calculer théoriquement un centre de gravité des charges selon la consommation puis dire : c'est là. Mais en réalité ça correspond pas à quelque chose de très pratique, donc on dit que le point d'injection devra se trouver entre Balbec et Brissol, en gros, dans ce secteur-là. » Ou encore: "C'est un centre mathématique si on tient compte du poids de la consommation géographiquement à un moment donné. On dit : il serait intéressant d'injecter de la tension à cet endroit-là ; parce que ça permet de couvrir les points de consommation par les réseaux $20000 \mathrm{~V}$ les plus courts. » Aucun argument technique ne justifiait donc qu'EDF implante le poste précisément sur Balbec: "On n'avait pas trop d'arguments pour dire que c'est vraiment à Balbec qu'il fallait le mettre. » L'emplacement de Balbec avait en quelque sorte été choisi par opportunité: le responsable d'EDF de l'époque avait pris contact de manière unilatérale avec la municipalité de Balbec pour rechercher un terrain susceptible de recevoir l'implantation du poste de transformation $63 \mathrm{kV} / 20 \mathrm{kV}$. De son côté, la municipalité de Balbec avait 
d'emblée réagi favorablement à ce projet en raison des rentrées financières que devait permettre l'application de la taxe professionnelle sur le poste source.

Mais l'emplacement est apparu au fil de la controverse de moins en moins pertinent en raison de ses mauvaises qualités géologiques ${ }^{6}$ et de ses difficultés d'acceptabilité, puisqu'il a réuni contre lui, outre le maire de Brissol, le comité et certains agriculteurs. Là aussi, le choix de l'emplacement participe à la production de connaissances qui s'accumulent au fil de la controverse. En l'occurrence, certains acteurs non-techniciens en acquérant une compétence technique ou, à tout le moins, en apprivoisant la technique ${ }^{7}$, ont réussi à dévier le projet. La détermination par EDF d'un centre de gravité initial (qui était, il est vrai, plus une zone qu'un point précis) s'est avérée insuffisante pour arrêter définitivement le projet, des acteurs ayant appris, entre temps, à se saisir de l'objet technique et à le rouvrir pour le modifier en fonction de leur point de vue et de leurs intérêts. Rien ne serait plus fallacieux en ce sens que de considérer qu'il y a d'un côté un objet stable (le centre de gravité déterminé par une série de calculs) et de l'autre, des luttes politiques extérieures aux enjeux techniques.

Pour l'emporter, chaque porte-parole a dû faire un effort pour tenir ensemble les impératifs d'EDF, des contraintes techniques et des enjeux socio-politiques. Il a fallu, par exemple, prendre en compte les exigences des défenseurs de l'environnement. Ces exigences entrent bien dans le compromis socio-technique final: l'emplacement initialement prévu pour le poste se trouvait en effet à l'extrémité de la zone intéressante pour le constructeur, côté mer ; après discussion, le poste a quitté le littoral, mais tout en restant cependant dans la zone intéressante pour le constructeur. La prise en compte de l'écologie a raccourci la ligne qui, en s'éloignant du littoral, a aussi permis à l'entreprise de réaliser une économie non négligeable sur l'ouvrage.

\section{La mise à l'épreuve de la notion de lieu}

21 La relecture empirique de la controverse sur la ligne à haute tension fait ressortir plusieurs éléments spatiaux dont nous soumettons les qualités et le rôle à discussion. Il ressort de l'analyse de la controverse sur cette LHT qu'aucune des entités spatiales concernées n'est un support passif de l'action en cours, ni une projection d'intentions socio-politiques. Ces entités spatiales qui sont tour à tour convoquées et invoquées lors des discussions, font partie de la controverse en tant que telle. La controverse a porté à un moment donné sur deux éléments simultanément : le calcul du centre de gravité des charges et l'emplacement de la ligne. La technique de son côté n'a rien pu trancher : par exemple, le recours aux chiffres et le recalcul du centre de gravité n'ont pas apporté un argument incontestable et définitif dans le débat, mais ont simplement déplacé l'objet de la discussion et permis de relancer la controverse sur un autre terrain. Dans la mesure où les élus, le comité, les riverains, etc., ont fortifié leur droit à la parole en acquérant un peu plus le pouvoir de s'emparer des questions techniques, l'objet technique a été reconfiguré. En fonction de la force des oppositions et des argumentations déployées par les divers interlocuteurs, la définition technique d'EDF a pu apparaitre plus contingente, moins «obligée». Le calcul du centre de gravité des charges fait par des acteurs différents permet en effet de requestionner le choix de l'emplacement du poste source et reconfigure l'espace existant.

22 Sur le plan méthodologique, si l'on évoque parfois en géographie des espaces (ou des milieux ou des territoires) donnés, une première question se pose immédiatement : que 
signifie exactement ce "donné »? Nous prendrons soin de nous décaler de cette perspective pour parler plutôt d'un espace ou d'un lieu "obtenu», en cherchant à thématiser ce qui l'a rendu explicite ${ }^{8}$. Mais comment nommer ces entités spatiales? Un premier argument en faveur de l'utilisation du concept de lieu est le problème des limites qui sont constamment soumises à discussion lors des controverses. C'est même une des caractéristiques principales de la controverse: changer les limites du dedans et du dehors, et la liste des éléments qui font ou non partie du problème (habitat, population, sol, etc.). L'idée de lieu contient cette dimension dynamique puisque selon D. Retaillé (1997), «ces mêmes lieux sont incertains, parfois éphémères (voir toutes les revendications possibles sur les territoires), et ne prennent pas sens par un contenu refermé sur lui-même. [...] Le lieu a comme propriété finale la co-présence » (Retaillé, 1997). Le lieu apparaît comme le résultat d'une solidarité éprouvée et non d'une solidarité de fait comme pour la proximité induite par l'appartenance implicite à un territoire (social, politique, administratif, etc.). Que fait-on dans une controverse si ce n'est de discuter des coexistences possibles, du monde commun dans lequel on souhaite vivre, lequel associe un ouvrage technique, un paysage que l'on ne souhaite pas défigurer, une alimentation électrique convenable etc. ?

À l'issue des débats, on établit des limites entre ce qui fait partie du problème et ce qui reste à l'extérieur montrant en cela que « ...les lieux se caractérisent aussi par le caractère sensible de leurs limites » (Lussault, 2003). Le lieu réalise (au sens de rendre réel) la mise en connexion d'une série d'éléments, il contribue à définir une identité, en associant des personnes, des objets, un sol particulier, une vue sur la mer, etc. Et la controverse contribue à produire des lieux : sans la LHT personne ne se serait mobilisé sur ce petit coin de terre pour se demander de quoi était composé le sous-sol présent sous leurs pieds.

Ainsi, comme le mentionne D. Retaillé (1997), « il ne suffit pas d'une proximité pour faire un lieu; il ne suffit pas non plus d'une présence observatrice de quelques ingrédients rapprochés. Il faut encore un déclenchement, une circonstance qui placent les sujets et les objets en position d'interaction». Une des conditions à la fabrication d'un lieu ne serait-elle pas ce que M. De Certeau, et à sa suite A. Micoud, appelle un événement, à savoir "ce qui est construit comme le point d'un commencement " (Micoud, 1992) ? En d'autres mots, cet événement pourrait fort bien ressembler à ce que M. Lussault (2000) nomme une situation.

L'idée de lieu nous semble pertinente car elle permet de décrire les dimensions spatiales de la controverse en passant avec succès les trois conditions requises pour son analyse. Elle tient compte du constant redimensionnement de la controverse, du côté éphémère et incertain dont vont être dotés les espaces au cours de celle-ci. Un lieu a une extension possible et n'est pas borné (dans le sens de délimité) a priori. Dans un lieu, les éléments hétérogènes « entrent » de façon libre et moins contrainte, puisque ce qui «fait lieu » est constamment redimensionnable. Les limites des endroits frappés par la controverse sont toujours à requestionner. En cela la notion de limite configurante proposée par M. Lussault (2003) est parfaitement adéquate, et la notion de lieu a la souplesse nécessaire pour en permettre l'analyse. Il se peut aussi que cette conception du lieu «en controverse » soit un complément intéressant aux diverses déclinaisons de cette notion proposée par B. Debarbieux (1995), même si ces derniers sont probablement moins pérennes.

Bien sûr, notre tour d'horizon est sommaire et nous n'avons fait qu'esquisser des pistes. Pour être plus complet, une autre série de questions devrait encore être posée à la notion 
de lieu, comme par exemple : cette notion suffit-elle pour suivre spatialement l'extension de la controverse? Pourquoi certains lieux ne s'assemblent pas à d'autres lieux de controverse? Et lorsqu'ils s'assemblent, comment qualifier l'extension des lieux controversés ? Ceci nous amène à poursuivre brièvement notre réflexion sur la notion de distance, pour aborder la question de l'extension/étendue de la controverse.

\section{Extension/Étendue de la controverse}

Il ressort de la controverse sur la LHT que la relation entre « la fabrique des lieux » et la distance est plus complexe qu'on ne pourrait le supposer. En effet, le déroulement de la controverse contribue à modifier constamment les distances entre les différentes parties prenantes (humains et non-humains) de celle-ci : un riverain souhaiterait que le poste s'implante ici et pas là ; en cela, il s'éloigne de ses voisins. En même temps, la LHT ne doit pas gêner la vue sur la mer et compromettre ce patrimoine touristique, etc.

Cette controverse a pour effet de rapprocher ou d'éloigner les différents éléments qu'elle concerne. Les mises à l'épreuve réalisées au cours des controverses définissent, pour un temps, le lieu et les distances effectives entre les êtres et les choses en indiquant à chaque fois ce qui fait partie ou non du problème. En ce sens, la position relative des phénomènes du même lieu semble avoir son importance dans les lieux controversés. Nous nous distinguons en cela de la définition de J. Lévy (1999), pour lequel, « dans une étendue, une distance est un ensemble d'attributs, d'informations caractéristiques d'un phénomène, d'une réalité, en fonction du degré de séparation qu'il/elle entretient avec d'autres phénomènes, d'autres réalités ".

Aussi, est-il possible de remarquer qu'à chaque fois qu'il y a création de distance, autrement dit, un déplacement pour reprendre les termes de B. Latour ${ }^{9}$, cette opération a un coût. Il y a un avant et un après, l'après étant toujours différent de l'étape précédente. Ce coût ne se chiffre pas nécessairement: il est simplement la traduction d'un effort. Nous sommes là aux fondements de l'acte géographique et de la spatialisation. En effet, pour « faire » un autre lieu ou pour co-construire un lieu, qu'il soit à controverse ou non, il y a un prix à payer pour le déplacement (au sens de création de distance ou de rapprochement). Il y a d'abord des êtres éloignés, et ceux-ci, au prix d'un effort important, vont créer des coexistences, construire des co-présences, créer des lieux. Cet effort, ce prix à payer, se traduit en termes de quantité de médiateurs à rencontrer : chaque être et chaque chose peut obliger à une transformation, à une bifurcation par rapport au projet initial. Cela a été le rôle du sol et de sa composition géologique par exemple. Celui-ci a contribué à transformer l'emplacement de la ligne. Cet effort, ce prix à payer, varie aussi en fonction des systèmes techniques à disposition, de l'état des connaissances scientifiques (quelles zones d'ignorance, quelles incertitudes, portant sur quoi ?) ainsi que des ressources que les individus mobilisent.

Enfin, signalons qu'un élément distingue les acteurs concernés par la ligne: pour les riverains, l'ouvrage est essentiellement localisé et c'est l'impact sur l'environnement qui compte; sur d'autres terrains, nous avons pu observer que sur une même ligne LHT les riverains mettaient tantôt en avant l'impact sur le patrimoine, tantôt l'influence éventuellement délétère des champs électromagnétiques sur la santé sans qu'apparaisse une communauté d'intérêts politiques tout au long de l'ouvrage. En revanche, pour les promoteurs du projet, la ligne et le poste ne sont que des éléments destinés à contribuer à la qualité de service de l'ensemble du réseau en alimentation électrique. Le lieu n'est donc 
pas le même selon les acteurs concernés : est-ce l'échelle géographique qui varie avec les acteurs impliqués $^{10}$ et comment rendre compte des effets de grandeur relative entre les différents éléments concernés par la controverse ? S'ouvre alors une question importante et controversée ${ }^{11}$ celle de savoir comment qualifier ces espaces où se mêlent des lieux, des aires et des réseaux.

\section{Conclusion}

31 Au terme de cette analyse, nous pouvons dire que les « entités spatiales » décrivent et en même temps participent à la construction des processus de configuration/ reconfiguration des collectifs (Latour, 1999). En effet, la controverse socio-technique et publique ne vise pas à débattre autour d'un objet qui reste stable. À l'issue des débats, tout a changé : les positions des acteurs (riverains et promoteurs), la ligne qui s'est raccourcie, les propriétés du sol se sont amoindries par rapport à l'idée que l'on s'en faisait, etc. C'est la raison pour laquelle on ne peut ni parler d'un « espace donné » qui serait en quelque sorte indiscutable, ni d'un espace sur lequel est projeté du travail et de l'information, selon la définition de C. Raffestin (1997), donc des valeurs qui seraient à jamais discutées. Les scientifiques ne sont plus seuls à discuter des faits et les sciences sociales ne définissent pas seules les valeurs a priori. De ce point de vue, notre étude de cas ne coïncide pas avec la définition de C. Raffestin (1997) : « Un territoire par rapport à un écosystème naturel n'est, en fait, rien d'autre que la projection de travail humain à l'aide de médiateurs - pratiques et connaissances - qui s'enracinent dans les sciences et les techniques. [...] Ce réagencement des écosystèmes naturels débouche sur une territorialisation. » Non seulement le rôle que jouent les sciences et techniques est plus souple que cette définition ne le laisserait supposer : il n'y a selon nous pas d'ancrage du territoire dans les sciences et techniques, mais plutôt un constant processus d'allerretour qui contribue à la fois à produire des formes spatiales, des sciences et des techniques; plus encore, la controverse n'est pas le résultat d'une projection mais est issue d'une interaction constante entre les éléments physiques et sociaux pour constituer in fine... ce qui semble davantage se rapprocher de la notion de lieu. Dans cette controverse, l'espace n'est pas seulement produit en fonction de schèmes collectifs d'essence sociale ${ }^{12}$, il est parfois co-construit par les habitants lesquels savent s'immiscer dans les objets scientifiques et techniques.

En effet, la dynamique de la controverse fait fi de ce partage entre les éléments physiques et sociaux. Pensons aux agriculteurs, au patrimoine ou encore au sondage géologique dans l'analyse des lignes à haute tension. Si le poste s'est finalement déplacé de Balbec à Brissol, ce n'est ni en raison de considérations « purement » techniques, ni en raison de considérations "purement " politiques, mais bien parce que des forces hétérogènes, mêlant intimement savoir technique et intérêts socio-politiques, ont pu être connectées : l'argumentation d'un maire recalculant un nouveau centre de gravité, des sondages géologiques, un travail de mobilisation mené par un comité d'opposants, etc. Pour l'emporter, chaque porte-parole a dû faire l'effort pour tenir ensemble les impératifs d'EDF, des contraintes techniques et des enjeux socio-politiques.

Pour revenir plus précisément à la notion de lieu, celle-ci peut se décliner selon trois acceptions différentes ${ }^{13}$ : l'acception géométrique définit le lieu comme point d'une surface, l'acception spatialiste définit le lieu comme une somme de qualités et de positions et enfin l'acception socio-anthropologique où le lieu est un emplacement qui 
fait sens dans la définition du rapport à l'espace d'un collectif. Il ressort de notre analyse que la fabrique des lieux s'inscrit dans un processus dynamique où il devient impossible de séparer ce qui relèverait de la seule inscription spatiale, ou des seules relations techniques ou sociales, prises séparément et déconnectées de l'espace où celles-ci se déroulent. La notion de lieu traduit selon nous cette capacité à tenir ensemble des éléments hétérogènes au sein de connexions qui se produisent en situation de controverse créant ainsi des délimitations qui ne sont jamais données d'avance. La controverse a besoin de lieux autant que les lieux ont besoin de controverses pour se fabriquer.

Notre dialogue entre une analyse de controverse et les concepts géographiques nous a permis de mieux saisir ce que nous pressentions de manière intuitive au début de notre discussion : d'une part les éléments spatiaux font partie intégrante de la controverse et sont davantage qu'un support passif de l'action. Ils participent à la controverse puisqu'ils agissent tout le long de l'évolution de la dynamique conflictuelle et ils (les lieux) en sont le résultat, ultime mais toujours provisoire lorsque la controverse diminue, s'arrête ou se clôture momentanément. D'autre part, les controverses ré-articulent les dimensions de nature et de culture, en les imbriquant et les entremêlant, rompant, en cela, avec les dichotomies existantes. Lorsqu'on cherche à définir ce qui caractérise les relations entre technique, espace et société en situation de controverse, on trouvera plutôt un brouillage de frontières que des limites claires et stables. Est-on là dans le «faire lieu », le «faire eikos» pour reprendre les mots de B. Latour, qui est si important pour toute pensée écologique, et corrolairement pour traiter les problèmes environnementaux? Si une controverse contribue à créer, à recréer ou à reconnaître des lieux ce qui est finalement une attention très ancienne, alors l'« espace » d'une controverse devient bien plus qu'un espace.

\section{BIBLIOGRAPHIE}

BolTANSKI (L.), 1990. - L'amour et la justice comme compétences, Paris, Métailié, 381 p.

CAllon (M.), LAscoumes (P.), BARTHE (Y.), 2001. - Agir dans un monde incertain : essai sur la démocratie technique, Paris, Seuil, $357 \mathrm{p}$.

CHARLIER (B.), 1999. - La défense de l'environnement : entre espace et territoire, thèse de doctorat, Université de Pau et des Pays de l'Adour, 753 p.

DEBARBIEUX (B.), 1995. - « Le lieu, le territoire et trois figures de rhétorique », Espace géographique, $\mathrm{n}^{\circ} 2$, p. $97-112$.

-, 2003. - « Territoire », dans LÉvy (J.), LusSAULT (M.) (dir.), Dictionnaire de la géographie et de l'espace des sociétés, Belin, Paris, p. 909-912.

Di MÉo (G.), 2000. - « Que voulons-nous dire quand nous parlons d'espace ? », dans LÉVY (J.), L ussault (M.) (dir.), Logiques de l'espace, esprit des lieux. Géographies à Cerisy, Belin, Paris, p. 37-48. 
FABUREL (G.) 2003. - « Le bruit des avions, facteur de révélation et de construction des territoires ", L'Espace géographique, $\mathrm{n}^{\circ}$ 3, p. 205-223.

LATOUR (B.), 1988. - «Comment redistribuer le grand partage », Revue du Mauss, n 1, p. 27-64.

-, 1989. - La science en action, Paris, La Découverte, 450 p.

-, 1999. - Politiques de la nature : Comment faire entrer les sciences en démocratie, Paris, La

Découverte, $382 \mathrm{p}$.

LATOUR (B.), SchWARTZ (C.), Charvolin (F.), 1991. - « Crise des environnements : défis aux sciences humaines ", Futur Antérieur, n 6, p. 28-56.

LECOURT (A.), 2003. - Les conflits d'aménagement : analyse théorique et pratique à partir du cas breton. Thèse de doctorat, Université de Rennes 2 Haute-Bretagne, 362 p.

LÉVY (J.), 1999. - Le tournant géographique. Penser l'espace pour lire le monde, Paris, Belin, 399 p. -, 2003. - « Territoire », dans LÉvy (J.), LuSSAULT (M.) (dir.), Dictionnaire de la géographie et de l'espace des sociétés, Paris, Belin, p. 909-912.

Lévy (J.), LusSAult (M.) (dir.), 2003. - Dictionnaire de la géographie et de l'espace des sociétés, Paris, Belin, $1033 \mathrm{p}$.

LiMOGES (C.), 1993. - « Expert knowledge and decision-making in controversy contexts », Public Understanding of Science, vol. 2, $\mathrm{n}^{\circ}$ 4, p. 417-426.

LoLIVE (J.), 1999. - Les contestations du TGV Méditerranée : projet, controverse et espace public, Paris, L'Harmattan, $314 \mathrm{p}$.

Lussault (M.), 2000. - «Action(s)! », dans LÉVy (J.), Lussault (M.) (dir.), Logiques de l'espace, esprit des lieux. Géographies à Cerisy, Paris, Belin, p. 11-36.

Lussault (M.), 2003. - « Lieu », dans LÉvy (J.), Lussault (M.) (dir.) Dictionnaire de la géographie et de l'espace des sociétés, Paris, Belin, p. 561-563.

Micoud (A.), 1992. - « Cadre, appropriation, qualification », dans GILBERT (F.), Micoud (A.), RouX (J.) (dir.), Socialisation, dynamiques locales et changement social, Actes du séminaire du GRS, Cahiers de recherche $n^{\circ} 10, p .119-132$.

OLLIVRo (J.), 1994. - Essai de modélisation d'une implantation ferroviaire : l'exemple du TGV Méditerranéen, thèse de doctorat, Université de Haute-Bretagne, $870 \mathrm{p}$.

RACine (J.-B.), RAFFESTiN (C.), RufFy (V.), 1980. - « Échelle et action, contributions à une interprétation du mécanisme de l'échelle dans la pratique de la géographie », Geographica Helvetica, vol. 35, $\mathrm{n}^{\circ}$ 5, p. 87-94.

RAFFESTIN (C.), 1997. - « Le rôle des sciences et des techniques dans le processus de territorialisation », Revue européenne des sciences sociales, t. XXXV, n 108, p. 93-106.

RÉMY (E.), 1995. - « Apprivoiser la technique, un débat public autour d'une ligne à haute tension ", Politix, $n^{\circ} 31$, p. 136-144.

RETAILLÉ (D.), 1997. - Le monde du géographe, Paris, Presses de la Fondation nationale des Sciences politiques, $283 \mathrm{p}$.

Soubeyran (O.), 2003. - « Milieu », dans LÉvy (J.), LusSAult (M.) (dir.), Dictionnaire de la géographie et de l'espace des sociétés, Paris, Belin, p. 617-619. 
TRICOT (A.), 1994. - « La prise en compte de la controverse dans l'aménagement du territoire : peut-on négocier le futur? ", Espaces et sociétés, n 74-75, p. 69-97.

WYNNE (B.), 1997. - « Controverses, indéterminations et contrôle social de la technologie. Leçons du nucléaire et de quelques autres cas au Royaume Uni », dans GODARD (O.) (dir.), Le principe de précaution dans la conduite des affaires humaines, Paris, INRA/Éditions de la Maison des Sciences de l'Homme, p. 149-178.

\section{NOTES}

1. Les événements que nous analysons se sont déroulés en Normandie entre 1989 et 1994. Ils sont décrit par E. Rémy (1995).

2. Si on abandonne la construction scientifique, il ne reste alors que les rapports de force, la pluralité des valeurs et des points de vue, autrement dit la seule construction sociale.

3. Pour des raisons de confidentialité, les noms de lieux sont fictifs. C'est aussi la raison pour laquelle, dérogeant à une pratique pourtant courante en géographie, nous ne pouvons accompagner cet article que d'une figure schématisée des lieux concernés.

4. Pour répondre à notre objectif de départ, nous nous éloignerons d'un raisonnement en termes de "points, lignes, surfaces ", car ce n'est pas le projet d'ensemble du tracé de la ligne qui nous intéresse dans le cadre de cet article. En effet, notre intérêt porte sur les lieux qui seront façonnés par l'implantation locale de la ligne et, plus précisément, par l'emplacement de son poste source.

5. Cette mise à l'épreuve s'inscrit en complément des recherches réalisées par A. Tricot (1994), dans la mesure où celles-ci abordent en priorité les notions d'espace, de milieu et de territoire dans ses analyses géographiques des controverses. C'est pourquoi nous avons choisi de nous concentrer avant tout sur la notion de lieu dans cet article.

6. Les sondages effectués sur le terrain initialement prévu ont montré que le terrain était inondable et extrêmement humide. En terme de traitement du sol, il aurait fallu décaper énormément d'argile et ramener beaucoup de remblai, ce qui aurait entraîné un surcoût important.

7. Comme le maire de Brissol, par exemple, se félicitant d'avoir réalisé des calculs jugés judicieux par les spécialistes.

8. Dans cette discussion, il faut mentionner également la notion de milieu. Nous pouvons noter que D. Retaillé (1997) propose un usage intéressant de cette notion, en articulant le milieu avec la notion de lieu, mais cet auteur s'empresse de préciser que cette acception est à écarter pour la connotation dont le terme est porteur : celui de milieu purement physique. C'est au nom de ce même argument que nous avons choisi de ne pas retenir cette notion dans notre discussion d'autant que ce terme est très utilisé par les sciences de l'écologie sans lien avec les interactions humaines. Il faut toutefois souligner que cette notion très critiquée traverse actuellement une phase de réhabilitation (Soubeyran, 2003).

9. Nous remercions infiniment B. Latour de nous avoir fait partager ses réflexions géographiques dans le cadre du séminaire Sogecer : Sociologie et géographie des controverses (environnementrisque), en particulier lors du séminaire à Pau (septembre 2002).

10. Pour une discussion sur les échelles géographiques, se reporter à l'article fondateur de J.-B. Racine et al., (1980).

11. Voir par exemple les définitions de J. Lévy (2003) et de B. Debarbieux (2003) dans le Dictionnaire de la géographie et de l'espace des sociétés.

12. Sur ce point se reporter notamment à l'article de G. Di Méo (2000). On pourrait encore mentionner les notions d'espace vécu, d'espace social et d'espace produit. Même si l'espace 
semble plus flexible, moins figé que le territoire, multidimensionnel, puisqu'il inclut le matériel et le symbolique, la dimension individuelle et sociale, les distances variables et les relations, il ne peut pas satisfaire complètement à l'étude socio-technique d'une controverse : la notion d'espace demeure essentiellement sociale, ce qui exclut les non-humains de l'action spatiale, réduits au rang de récepteurs de l'action, incapables de la relancer.

13. Nous remercions B. Debarbieux d'avoir attiré notre attention sur ce point dans une version préliminaire de ce texte.

\section{RÉSUMÉS}

En nous appuyant sur une étude de sociologie des sciences et des techniques portant sur une controverse autour d'une ligne à haute tension située en Normandie, nous avons l'intention de montrer que l'espace concerné contribue à remodeler et orienter la controverse, autant que celle-ci forge et modifie les espaces qu'elle touche. Avec la progression de l'analyse, la notion de lieu nous a semblé mieux adaptée à rendre compte de la complexité et de la variabilité de la géographie de la controverse. La notion de lieu traduit selon nous cette capacité à tenir ensemble des éléments hétérogènes dont les délimitations ne sont jamais données d'avance.

This paper studies how the idea of space (such as involved in a controversy about the location of a hight-tension power line in Normandy) contributes to remodeling and reorienting the controversy, as much as the controversy remodels and orients the space. The base of the study was a sociological approach to science and technology. Thought, as our analysis progresses, the idea of place (instead of space) seems better adapted to explain the complexity of the geography of the controversy. The place translates the capacity to contain all of these heterogeneus elements and to re-organize them.

\section{INDEX}

Mots-clés : acteurs, controverse, lieu, ligne à haute-tension, territoire

Keywords : actors, controversy, high-tension line, place, territory

Index géographique : France, Normandie

\section{AUTEURS}

\section{VALÉRIE NOVEMBER}

Département de Géographie - Université de Genève

\section{CRISTINA D'ALESSANDRO-SCARPARI}

Geology and Geography Department - West Virginia University (WV) USA, cristinad@geo.wvu.edu 


\section{ÉLISABETH REMY}

Sociologue, UMR SAD-APT - INRA, elisabeth.remy@inapg.inra.fr 